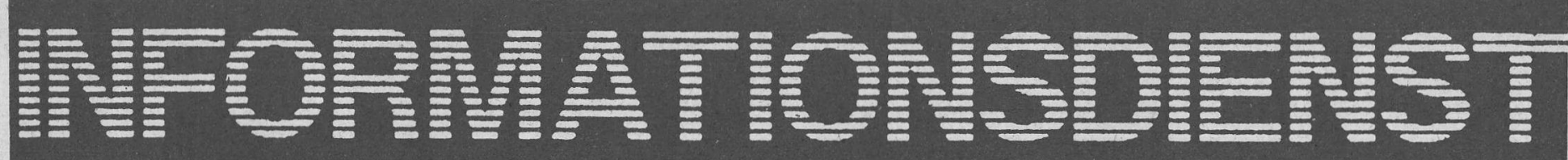

MSTITUT FUR OKOLOCISCHE MITSCHA TSFONSCHUNG

VEREINIGUNG FUR OKKOLOGISCHE VIRTSCHAFTSFORSCHUNG

\section{Energieeinsparung - Thema der Zukunft}

Was in der Bundesrepublik noch undenkbar erscheint, in Dänemark ist es möglich: Die Vereinigung der Elektrizitätswerke veranstaltete die erste internationale Konferenz über Energieeinsparung mit dem Schwerpunkt Elektrizitätseinsparung 1.-4. September in Kopenhagen mit fast 500 Teilnehmern aus aller Welt. Ebenfalls zum Thema Energieeinsparung veröffentlichte die IEA (International Energy Agency) im Frühjahr eine umfangreiche Studie (IEA/OECD, Energy Conservation in IEA Countries, Paris 1987).

Nach der Eröffnung durch den konservativ-liberalen dänischen Energieminister berichtete L. Philip Martin, Senior Vice President der Southern California Edison Company, USA von den erfolgreichen Bemühungen der Elektrizitätswerke in Kalifornien den Elektrizitätsverbrauch $\mathrm{zu}$ drosseln und erneuerbare Energien zu fördern. Aber - und das war vielleicht der interessanteste Punkt - er berichtete auch freimütig, $\mathrm{da} ß$ der Anreiz zu Energieeinsparungen in den letzten beiden Jahren verschwunden sei. War es nämlich nach den Ölpreisschocks in den 70er Jahren für die Elektrizitätsunternehmen selbst von Vorteil, den Anstieg des Elektrizitätsverbrauchs zu drosseln, da die marginalen Grenzkosten einer produzierten Stromeinheit über den Durchschnittskosten lagen, so hat sich dieses Verhältnis nach den Ölpreissenkungen genau umgekehrt. Daher bestehe - auch weil das Angebot von unabhängigen Stromerzeugern ständig zunehme deren Überschuß sie gezwungen seien abzunchmen - kein Interesse mehr an Energieeinsparung. Die Bemühungen der kalifornischen Elcktrizitätswerke seien nun darauf gerichtet, zilar den Spitzenlastverbrauch nicht weiter ansteigen zu lassen, aber gleichzeitig die Täler im Stromicrbrauchsniveau aufzufüllen. - Lernen läßt ich hieraus, daß das Anliegen der Energieein sparung nicht frei schwankenden Markpreisen uberlassen werden sollte.

Den zweiten Vortrag hielt Suzanne Frigren. ( $\mathrm{m}$ welt-u. Energieministerium Schweden, dic u.a. über die ersten Ergebnisse der nach dem Tschernobyl-Unfall eingerichteten Energiekommission berichtete: Ein Ausstieg aus der Kerneenergie wird danach vorauss. nicht vor 1999 möglich scin. Ein weiterer sehr interessanter Vortrag von Tak a nomi Tohmatsu, Kansai Electric Power, Japin schilderte die erfolgreiche Anwendung von progressiven Elektrizitätstarifen zur Reduzierung des Elektrizitätsverbrauchs in Japan.
Am Nachmittag des 2. Tages wurden Untersektionen gebildet, deren größte sich mit dem Thema „Tarife, Abgaben, Finanzierung und $\mathrm{Zu}$ schüsse zu Energieeinsparungen" befaßte. Aus der Fülle der Vorträge sei nur die detaillierte Analyse von Energiesteuern von Tom Togsverd, Finanzministerium Dänemark, herausgegriffen. Er beschrieb den bemerkenswerten Versuch Dänemarks, die Verbraucherpreise für Energie trotz Ölpreisverfall konstant zu halten. Damit ist die dänische Energieabgabe gegenwärtig eine der höchsten der Welt. Allerdings sind die privaten Unternehmen von der Abgabe befreit. Man würde jetzt erwarten daß die dänischen Elektrizitätspreise ebenfalls extrem hoch sein müßten. Dem ist jedoch nicht so:

Tabelle:

Internationaler Vergleich von Elektrizitätssteuern und -preisen (Dkr/kwh). Stand 1986 - incl. V.A.T.

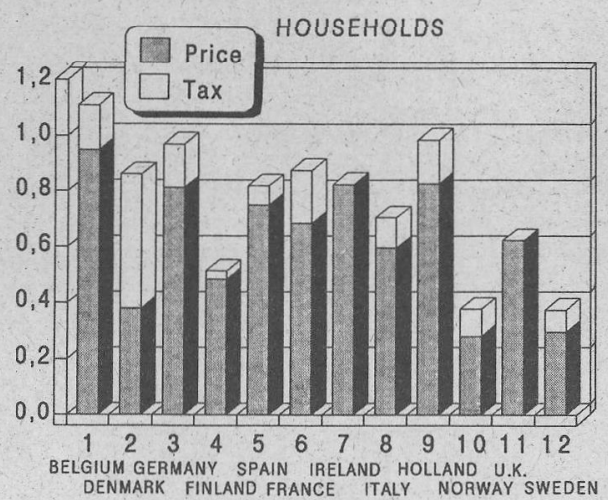

Ouclle: First Intcrnational Srmpocium on Fnergy S: vings, Kopenhagen 1487, Vortrag Tom Togsverd, Taxes to the State, Kongreßmaterialen

Fortsetzung S. 2
4. $-6.12 .87:$

IÖW-Jahrestagung

„Wirtschaftsethik und ökologische Wirtschaftsforschung"

Die Rede von der Wirtschaftsethik ist in Mode gekommen. Philosophen und Theologen, Ökonomen und Soziologen besuchen oder organisieren Tagungen zum Thema, lesen oder schreiben darüber. Praktische Bemühungen um die Einlösung gesellschaftlicher Verantwortung von Unternehmen und Unternehmenskultur ordnen sich ebenso zu wie die allgemeine Frage, wie denn ein mit seiner Verselbstständigung viel Schaden und Zerstörungen anrichtendes Subsystem Ökonomie gesellschaftlich gebändigt und vernünftig in einen Diskurs um erstrebenswerte Ziele eingebunden werden könnte.

Die ökologische Herausforderung an die Gesellschaft im allgemeinen, die Ökonomie im besonderen kommt unserer Meinung nach bei dem bisherigen wirtschaftsethischen Diskurs zu kurz.

Darum führt das IÖW seine Jahrestagung 1987 vom 4.-6. Dezember mit dem programmatischen Titel: „Wirtschaftsethik und ökologische Wirtschaftsforschung" durch.

Referieren und ihre Konzeptionen von Wirtschaftsethik zur Diskussion stellen werden u, a.:

Prof. Dr. Klaus-Michael Meyer-Abich über praktische Naturphilosophie,

- Prof. Dr. Burkhard Strümpel über Erosion der Arbeitsethik und die Folgen,

- Prof. Dr. Peter Ulrich und Prof. Dr. Horst Steinmann zur Relevanz der kommunikativen Ethik und des Konstruktivismus für wirtschaftsethische Fragestellungen.

Dr. Eberhard Seifert (Hamburg) wird seitens des IÖW den Überlegungsstand hinsichtlich einer ökologischen Wirtschaftsethik vortragen. Nähere Informationen und Anmeldung zur Tagung über das IÖW. 


\section{Fortsetzung von $S .1$}

Die Elektrizitätpreise liegen in Dänemark trotz sehr hoher Besteuerung nur an 5.Stelle in Europa. Auffallend niedrig sind die dänischen Erzeugungskosten verglichen mit Ländern, die mit „billigem Atomstrom" operieren wie Frankreich und die Bundesrepublik.

$\mathrm{Zu}$ den Themen Wärmeplanung, erneuerbare Ressourcen und dezentrale Kraft-Wärme-Kopplung wurden am 3. Tag Untersektionen gebildet. Das dänische System der Wärmeplanung beschrieb Helge Ørsted Pedersen, Energieministerium Dänemark. Seit 1979, dem Jahr des „Heat Supply Act", gibt es eine zentrale Wärmeplanung in Dänemark mit beachtlichen Erfolgen bei der Energieeinsparung im Raumwärmesektor. Zum Beispiel hat sich der Prozentsatz der Anschlüsse an das Fernwärmenetz von $23 \%$ im Jahr 1975 auf 43 \% im Jahr 1985 erhöht. 1995 werden voraussichtlich $70 \%$ aller Haushalte an kollektive Netze angeschlossen sein.

Den Abschluß der Tagung bildete eine Podiumsdiskussion mit einer heftig geführten Debatte, inwieweit der Staat in die Marktprozesse eingreifen soll. Nach dem „Brundtland-Report" 1987, so Prof. Niels Meyer, Dänemark, sei die Reduzierung des Energieverbrauchs um $50 \%$ in den industrialisierten Ländern die einzige Chance für den Nicht-Zusammenbruch des globalen Ökosystems im nächsten Jahrhundert.

In der ebenfalls bemerkenswerten IEA-Studie wird festgestellt, daß die Energieeinsparung eine bedeutende Komponente der internationalen Energiepolitik geworden ist. Gründe für die zukünftige Bedeutung der Energieeinsparungspolitik werden hervorgehoben: Die Energieeinsparung weite die Verfügbarkeit über erschöpfbare Energieressourcen aus, vermindere zweitens das Gewicht zukünftig erwarteter Energiepreissteigerungen und reduziere drittens die Umweltbelastungen. Außerdem seien Investitionen in Energieeinsparung oft von einer höheren Rendite gekennzeichnet als Investitionen in Vergrößerungen des Energieangebots und seien zudem in kleinen Schritten durchführbar.

Die Erfolge in der bisherigen Energieeinsparung sind beträchtlich: In der gesamten IEA Region ist die Energieintensität (gemessen als Energieinput pro produzierter Sozialprodukteinheit) zwischen 1973 und 1985 um $20 \%$ gefallen. Dies sei insb.auf die gestiegenen Energiepreise, aber auch auf unterstützende Maßnahmen von staatlicher Seite zurückzuführen. Trotzdem verbleibe ein großes Potential für weitere Energieeinsparungen. Selbst wenn nur die gegenwärtig rentablen Maßnahmen bis zum Jahr 2000 durchgeführt würden, könnte die Energieeffizienz dann um mehr als $30 \%$ höher sein als gegenwärtig.

Bemerkenswert auch die Skepsis der Verfasser gegenüber dem Marktmechanismus: Nur in der Theorie, so die IEA Studie, gewährleiste der Markt eine optimale Allokation der Ressourcen im Energiebereich, einschließ1. eines angemessenen Bemühens um Energieeinsparung. In der Praxis sei dies aus einer Reihe von Gründen nicht gewährleistet: Z.B. reflektierten die Marktpreise vor allem die kurzfristigen Einflüsse und weniger die langfristige Entwicklung. Außerdem würden die Energiepreise typischerweise nicht die externen Kosten und Nutzen miteinbeziehen, insbes. nicht die Umweltschutz- und Sicherheitskosten. Aus diesen Gründen empfiehlt die IEA staatliche Maßnahmen stärker zu forcieren, z.B. - die Unterstützung von Individuen, privaten Firmen und anderen Organisationen, die in Energieeinsparungsaktivitäten tätig sind.

- Die Möglichkeit erhöhter Energiesteuern als Anreiz zur Energieeinsparung stärker zu nutzen. Bisher werde dies nur in wenigen Ländern explicit versucht, so in Dänemark, Portugal und Schweden.

- Demonstrationsprogramme für bestehende energieeinsparende Technologien sollten gefördert und die Forschung und Entwicklung von Neuen Technologien forciert werden. Dies schließe die sozio-ökonomische Forschung ein, um die Formulierung effizienter Energiepolitik voranzutreiben (!)

Es bleibt zu wünschen, daß die internationale Diskussion auch die Energiepolitik der Bundesrepublik ein wenig zu bewegen vermag.

Sabine Spelthahn, Berlin

\section{Billiger Atomstrom?}

Daß Kernkraft billiger sei, wird von deren Befürwortern gern behauptet. Außer Modellrechnungen mit fragwürdigen Annahmen fehlen hierfür aber die Belege. Die tatsächl. Preise lassen einen solchen Kostenvorteil jedenfalls nicht erkennen. Weder in der Bundesrepublik, wo „Kernenergieländer" wie Hamburg oder Baden-Würtemberg besonders hohe Strompreise haben, noch im internationalen Vergleich (s. Darstellung)

Bezieht man in diesen Vergleich die Strompreise der Haushalte ein, so zeigt sich bei den Atomländern Frankreich und Belgien, daß die Strompreise für Industriekunden massiv durch die Haushalte subventioniert werden. Ähnliches gilt für die Bundesrepublik Deutschland und Spanien. Der Mythos vom billigen französischen Atomstrom verblaßt zusätzlich vor der hohen Verschuldung des staatlichen Elektrizitätsversorgungsunternehmens (EDF): 213 Mrd. frs. im Jahre 1985 - das 1,6 fache des gesamten Umsatzes!

Schweden und die Schweiz haben zwar - bei hohem Kernenergieanteil - relativ niedrige Strompreise. Aber sie haben auch einen besonders hohen Anteil an Elektrizität aus Wasserkraft. Und die ist unbestritten billig, was sich in Norwegen und Neuseeland besonders günstig auswirkt. Unter den Durchschnittspreisen der westlichen Industrieländer liegt auch das Kohleland Australien. In Dänemark wiederum ist der ohne Kernkraft erzeugte Haushaltsstrom teurer, weil er massiv besteuert wird. Dem liegt eine energiepolitische Absicht ebenso zugrunde wie dem hohen Strompreis in Japan: Er ist dort auch für die Industrie weitaus höher als bei den Weltmarktkon-
Kernenergieanteil an der

Stromerzeugung und Strompreisen in westlichen Industrieländern 1985

\begin{tabular}{lcc} 
Land & $\begin{array}{c}\text { Kernnergie- } \\
\text { anteil in v.H. }\end{array}$ & $\begin{array}{c}\text { Strompreis(US- } \$ \text { ) } \\
\text { Haushalte/Industrie }\end{array}$ \\
\hline a) Länder mit Kernenergie: & \\
Frankreich & 65,1 & $0,085 / 0,037$ \\
Belgien & 60,4 & $0,101 / 0,043$ \\
Schweden & 42,8 & $0,040 / 0,029$ \\
Schweiz & 39,9 & $0,059 / 0,047$ \\
Finnland & 38,5 & $0,052 / 0,041$ \\
BR Deutschland & 30,8 & $0,082 / 0,047$ \\
Japan & 23,7 & $0,123 / 0,091$ \\
Spanien & 22,0 & $0,086 / 0,046$ \\
Großbritannien & 20,5 & $0,067 / 0,046$ \\
USA & 15,5 & $0,078 / 0,052$ \\
Kanada & 13,2 & $0,037 / 0,023^{1}$ \\
Niederlande & 6,2 & $0,087 / 0,042$ \\
Italien & 3,8 & $0,077 / 0,059$ \\
b) Länder ohne Kernenergie: & \\
Australien & 0,0 & $0,052 / 0,031$ \\
Dänemark & 0,0 & $0,086 / 0,046$ \\
Griechenland & 0,0 & $0,072 / 0,050^{2}$ \\
Irland & 0,0 & $0,088 / 0,062^{1}$ \\
Luxemburg & 0,0 & $0,067 / 0,039^{1}$ \\
Neuseeland & 0,0 & $0,029 / 0,022$ \\
Norwegen & 0,0 & $0,039 / 0,014$ \\
Osterreich & 0,0 & $0,085 / 0,040^{1}$ \\
Portugal & 0,0 & $0,075 / 0,056$ \\
Durchschnitt: & & $0,071 / 0,044$ \\
\hline & & $1=1984$ \\
\hline & & \\
\hline & & \\
& &
\end{tabular}

Quelle: Forschungsstelle für Umweltpolitik an der Freien Universität Berlin, 1987. Datenbasis: Internationale Energieagentur (IEA)

kurrenten. Dafür regt er im Bereich der Elektrotechnik neue, sparsamere Technologien an. Und das ist durchaus erwünscht.

Martin Jänicke, Berlin
Vereinigung für ökologische Wirtschaftsforschung (VÖW) e.V.

Konto: 790010070 Sparkasse Berlin, BLZ 10050000 Vorstand: Dr. Mechthild Oechsle, Prof. Dr. Eberhard Schmidt, Sabine Spelthahn, Prof. Dr. Helmut Spitzley, Antje Theißen

Institut für ökologische Wirtschaftsforschung (IÖW) GmbH

Konto: 790010135 Sparkasse Berlin, BLZ 10050000 Beirat: Dr. Marlene Kück, Dr. Irene Schöne, Prof. Dr. Johannes Berger, Dr. Eckart Hildebrandt, Dr. Carlo Jaeger-Weise, Prof. Dr. Martin Jänicke, Dr. Christian Leipert, Dr. Joachim Müller, Dr. Reinhard Pfriem, Dr. Eberhard K. Seifert, Prof. Dr. Burkhard Strümpel, Dr. Otto Ullrich, Dr. Helmut Wiesenthal 
(c) 20I0 Authors; licensee IÖW and oekom verlag. This is an article distributed under the terms of the Creative Commons Attribution Non-Commercial No Derivates License (http://creativecommons.org/licenses/by-nc-nd/3.o/), which permits unrestricted use, distribution, and reproduction in any medium, provided the original work is properly cited. 